\title{
Nurses' use of the Internet and social media: Does age, years of experience and educational level make a difference?
}

\author{
Debra M. Wolf ${ }^{* 1}$, John Wenskovitch ${ }^{2}$, Bonnie B. Anton ${ }^{3}$ \\ ${ }^{1}$ Chatham University, Pittsburgh, PA, United States \\ ${ }^{2}$ Allegheny College, Meadville, PA, United States \\ ${ }^{3}$ University of Pittsburgh Medical Center, St Margaret Hospital, Pittsburgh, PA, United States
}

Received: August 10, 2015

DOI: $10.5430 /$ jnep.v6n2p68
Accepted: October 15, 2015

Online Published: November 2, 2015

\begin{abstract}
Introduction: Does age, level of education, or years of experience influence nurses' ability to use the Internet safely? As visionary nurse leaders, one has a responsibility to support nurses' level of e-Professionalism and use of the Internet to extend virtual services to patients and the community. Numerous articles caution nurses on the legal, ethical, and moral concerns in using the Internet, but few studies research nurses' use of the Internet based on age, years of experience, and level of education. Objective: To explore nurses' use of the Internet and social media.

Methods: Nurses were invited to complete an Institutional Review Board (IRB) approved 51 questions electronic survey.

Results: Nurses shared information regarding use of the Internet. Statistically, significant findings were noted based on one's age, educational level, and years of experience.

Conclusions: Nurse leaders need to assess and educate nurses in using social media for personal and professional needs in order to enhance the delivery of virtual services.
\end{abstract}

Key Words: Social media, Internet, Health information, Nurses, Technology

\section{INTRODUCTION}

With the continual expansion of the World Wide Web (WWW), use of the Internet to seek or share information virtually has become common practice. Today, individuals freely post information to social media sites about their daily routines, personal experiences, current locations, and adding photos and videos to validate the experience. Using personal computers, smart phones, or tablets, individuals now have the technology that allows them to communicate globally within seconds of entering a key stroke. Using innovative technology and virtual platforms, health professionals have an opportunity to pursue the next generation of how health information is shared and how virtual health-related care can be delivered. This level of virtual care requires health professionals to be educated, for their ethical and legal responsibilities are further influenced. Over the past few years, articles have been written alerting health professionals on the legal, ethical, and moral concerns regarding the use of social media in their professional role. ${ }^{[1-5]}$ Unfortunately, little research has been published that explored nurses' use of social media personally and professionally in regards to their age, level of education, and years of experience. This

*Correspondence: Debra M. Wolf; Email: dwolf@chatham.edu; Address: Chatham University, Woodland Road, Pittsburgh, PA, United States. 
article provides the results of an Institutional Review Board (IRB) approved exploratory study that examined nurses' use of the Internet, electronic devices, and the use of social media personally and professionally.

\subsection{Background}

Over the past decade, nursing has transformed into a profession supported with numerous types of innovative technology to care for patients in various clinical settings. Today, nurses are using electronic health records (EHRs) to document patient care; a variety of technology to capture vital signs, pulse oximetry and glucose checks; clinical decision support, rules and logic to guide clinical decision-making; and bar code scanning to administer medications, treatments, and/or collection of specimens. This transformation reflects how nurses are becoming comfortable in using technology to support their professional practice.

Within one's personal life, nursing professionals are also expanding their use of technology. Owning a smart phone, tablet, computer, or eReader connected to the Internet and various social media sites has become common practice for many individuals. Unfortunately, the use of technology and the Internet by nursing professionals has raised concerns regarding legal, ethical, and moral responsibilities, with one's professional roles becoming blurred with their private life. ${ }^{[1-5]}$

\subsection{Literature review}

A review of the literature indicated the need for nursing professionals to advance their knowledge in using the Internet for professional needs. Cain ${ }^{[6]}$ discusses the concept of eProfessionalism, a term used to define the legal and ethical implications of using online communication (social media) by health professionals. Nurses must understand how to separate their personal and professional life virtually when using the Internet and social media to communicate with peers, patients, family, and friends. In nursing school, students are educated on the nursing Code of Ethics, but today, they must also be educated in understanding the role e-Professionalism plays on their nursing career.

Spector and Kappel ${ }^{[5]}$ identified seven categories (see Table 1) of errors that occur when nurses have a poor understanding of e-Professionalism and do not separate their personal life from their professional role. The authors share three actual scenarios that clearly present situations in which nurses lacked e-Professionalism. For example, in one scenario the author shares how a nurse refers to the disabled child she cares for as her "little handicapper" on a public newspaper's blog, noting the child's age and use of wheelchair (para. 9). In a second scenario the implications of forwarding an inappropriate email among nursing personnel of a patient in a hospital gown led to law enforcement involvement with nurses being placed on administrative leave (para. 10). Finally, a scenario was presented illustrating how a student nurse was expelled from a nursing program, the nursing program was no longer allowed to have clinical rotations in the facility, and the hospital faced HIPAA violations, all due to posting a picture on a social media site of a pediatric patient (para. 11). Nursing professional organizations are slowly moving forward in alerting nurses to be more vigilant in their level of e-Professionalism. Currently there are two resources nurses can use to guide their level of e-Professionalism. The American Nurses Association ${ }^{[7]}$ published ANA Principles for Social Networking and the Nurse which offers six guiding principles (see Table 2), and a white paper by the National Council of State Boards of Nursing ${ }^{[8]}$ that provides guidance on how to avoid problems when using social media, focusing on confidentiality and privacy myths and misconceptions.

Table 1. Categories of errors when nurses misuse social media

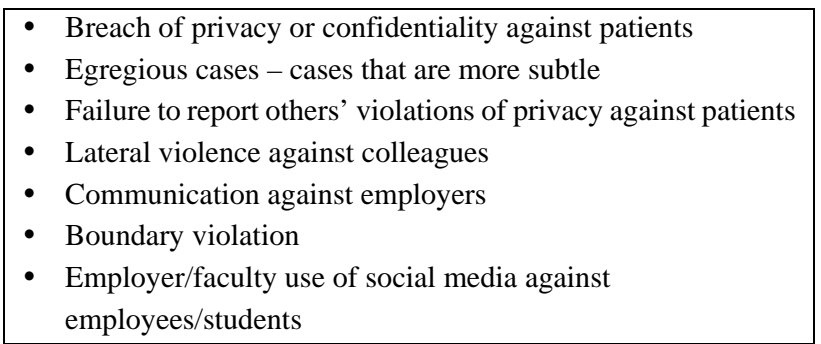

Note. Categories taken from Spector \& Kappel (2012)

Table 2. The ANA principles for social networking and the nurse

1) Nurses must not transmit or place online individually identifiable patient information.

2) Nurses must observe ethically prescribed professional patient-nurse boundaries.

3) Nurses should understand patients, colleagues, institutions and employers may view postings.

4) Nurses should take advantage of privacy settings and seek to separate personal and professional information online.

5) Nurses should bring content that could harm a patient's privacy, rights, or welfare to the attention of the appropriate authorities.

6) Nurses should participate in developing institutional policies governing online conduct.

Note. Principles taken from ANA (2011) 
As the world continues to openly embrace innovative technology and the use of the Internet, healthcare professionals and organizations must follow. Consumers are turning to the Internet to seek health information, to find a provider, to explore treatment options, or to confirm diagnosis. ${ }^{[9]}$ Establishing a virtual presence allows nurses and healthcare organizations to extend their services virtually, and to meet the needs of the consumer and other health professionals. Today, there are several nursing professions and health organizations that successfully use the Internet and social media to extend their services virtually. ${ }^{[4,10-12]}$ Examples of virtual services include 1) educational programs, 2) community health networking groups, 3) health resources and 4) information regarding best practice. Nurses and health care organizations need to understand how a health professional's age, level of education, and years of experience influence their ability to use the Internet in an e-Professional manner. As visionary leaders, nurse leaders need to support the use of the Internet to extend virtual services, but first they need to understand their employee's knowledge level and use of the Internet for personal and professional needs. Results of an exploratory study reveal that one's age, years of experience, and level of education may influence their level of e-Professionalism.

\section{RESEARCH METHODOLOGY}

In 2014, an IRB-approved exploratory study was completed to assess nurses' use of technology, the Internet, and social media for personal and professional needs. IRB approval was obtained through Chatham University's IRB. Nurse leaders from a national organization, three large tertiary community hospitals, and a community leader with a strong web presence, invited nurses to participate via email communication, posting on web sites and through paper flyer communication. Nurses were asked to complete a self-developed electronic questionnaire that contained 51 multiple choice questions (using $\mathrm{a}, \mathrm{b}, \mathrm{c}, \mathrm{d}$ options) and fill in questions. The questionnaire was reviewed by informatics nurse specialists and curriculum development academic experts leading curriculum in nursing informatics confirming that the content, questions, and format were appropriate and valid. The questionnaire was divided into four sections: demographics, Internet usage, use of electronic devices, and how nurses assess their patients' use of social media.

Initially in 2013, a pilot study was conducted to assess if this format and process would be effective. Approximately 760 nurses participated in the pilot and completed the survey. The researchers evaluating the pilot study's methodology found the method of invitation and completion of electronic survey was efficient and effective, resulting in no changes to the methodology for the larger study. Upon review of the preliminary data, the researchers were confident the survey was appropriate and valid, requiring no formal changes, other than minor typo errors found. Once confirmed, the pilot study was extended into a larger scale study. Data were compiled and analyzed using Excel 2010, and evaluated using chi-square tests. Statistical significance was determined using a $p$-value of .05 .

\section{Invitation to participate}

Initially, nurse leaders were introduced and invited to take part in the study through various forms of communication. The researchers announced the study at professional organized conferences, inviting various nurse leaders to contact them if interested. Next, through networking opportunities, the study was shared with health professionals of various leadership positions. A total of five nurse leaders agreed to participate in the study: one from a national nursing organizations with a membership of $>5,000$ nurses, three from tertiary community hospitals in the states of Pennsylvania, New Jersey, and Texas; and one community leader. The community nurse leader was a well-established nursing professional with over 25 years' experience as a nurse, nurse educator, and nurse executive. This individual has been identified through the literature and other well-established organizations as a leading authority in nursing. Currently, the leader is the CEO and President of her own company, which has a strong web presence, guiding and leading nurses to a higher level of professionalism.

The researchers scripted an original email, outlining the study's purpose and inviting nurses to take part in the study by completing an electronic survey, by clicking on the active URL hyperlink embedded in the email. The email was forwarded one time to the five nurse leaders asking them to forward to their nursing personnel. The researchers had no actual contact with any of the participants who completed the surveys. There was no monetary or other incentive offered to participate in study.

\section{RESUlts}

Approximately 7,881 nurses were invited to take the survey, with a $15 \%$ response rate achieved $(n=1,196)$. Participants were not forced to complete each question, a decision made by researchers to reduce any type of dissatisfaction in completing survey in a timely manner due to the number of survey questions. All survey data was used in the statistical analysis, regardless of whether or not an individual participant completed all 51 questions. In cases where a participant only partially completed the survey, the questions that they did not respond to were not factored into the analysis. Therefore, 
a number of questions were analyzed with fewer than 1,196 responses.

The average responder was a Caucasian female, aged 46-55, with a BSN, employed full time with 25 years of experience. The results of each question were reviewed individually, but a more detailed analysis was conducted exploring possible relationships in regards to age (tested in three groups with ages split at 55, 45, and 30), highest nursing degree achieved (tested in groups divided by graduate degree, Bachelor degree, Associate degree, and diploma), and years of experience (tested in groups split at 10, 20, and 30 years of experience). A summary of participant's age, highest nursing degree and years of experience is provided in Table 3 . When analyzing the data, several statistically significant findings were noted when reviewing six particular questions on the survey. Below is a summary of the findings based on nurses' response to the six question and to other questions which were not statistically significant.

Table 3. Summary of participant's age, highest nursing degree, and years of experience

\begin{tabular}{ll}
\hline Items & N (\%) \\
\hline Age: & $85(7.1 \%)$ \\
-30 & $237(19.8 \%)$ \\
$31-45$ & $303(25.3 \%)$ \\
$46-55$ & $517(43.2 \%)$ \\
$55+$ & $54(4.5 \%)$ \\
No Response & \\
Highest Nursing Degree: & $130(10.9 \%)$ \\
Diploma & $285(23.8 \%)$ \\
Associate & $493(41.2 \%)$ \\
Bachelor & $228(19.1 \%)$ \\
Graduate & $60(5.0 \%)$ \\
No Response & \\
Years of Experience: & $141(11.8 \%)$ \\
$<10$ & $150(12.5 \%)$ \\
$10-20$ & $177(14.8 \%)$ \\
$20-30$ & $319(26.7 \%)$ \\
$30+$ & $409(34.2 \%)$ \\
No Response &
\end{tabular}

\section{Q1: Nurses who received formal education on how to use the Internet safely}

When answering the question regarding whether or not they received formal education on how to use the Internet safely, nurse's age and years of experience had statistically significant results. Results reflected nurses in the $\leq 30$ age group were more likely to have received educational training in how to use the Internet safely than those in the $\geq 31$ age group $(p=.0070)$. Likewise, nurses who reported 10 or fewer years of experience were also more likely to have received edu- cational training than those with $\geq 11$ years of experience $(p=.0059)$. These findings support the idea that Internet safety is taught more today in schools of nursing than in previous years. Given how long the Internet has been in existence, and with its rapid expansion in recent years, these findings are conceivable. The conclusion supports the belief that Internet safety training should be recommended for senior nurses (referring to nurses with more seniority in years working, who have been out of school for several years), and as a reminder for younger nurses to expand and lead health services virtually. Despite the age of nurses, Internet safety is an important component of institutional educational in-services.

\section{Q2: Nurses use guidelines to assess the quality of a web- site}

Interestingly, nurses' age and years of experience were influential factors in how they responded to the question that explored the nurse's use of formalized guidelines to assess the quality of a website. Formal guidelines were defined as an organized process to determine or assess the quality of a website by identifying, who the authors (publishers) of the website were; what was the date of the publication posted on the website; who was sponsoring the website; and what was the quality of the content on the website. Nurses who reported an age of $\geq 31$ years were more likely to use guidelines to assess the quality of a website than those who reported an age of $\leq 30$ years $(p=.0012)$. In addition, statistical findings were noted in relation to years of experience. Nurses who reported $\geq 11$ years of experience were more likely to use guidelines to assess the quality of a website than those with $<10$ years of experience $(p=.0057)$. This finding may suggest senior nurses are aware of their lack of knowledge in how to use the Internet safely and resort to using guidelines to assist them. Younger nurses with less experience may feel more confident in using digital technology and the Internet and don't feel they need guidelines.

\section{Q3: Nurses who suggest websites}

When asked if nurses suggest websites to their clients, employees, patients, and/or family members to review health information, several interesting statistically significant results were found in every age breakdown. Nurses who reported an age of $\geq 55$ were more likely to suggest websites than those who reported an age of $\leq 54(p<.0001)$. Similarly, nurses who reported an age of $\geq 46$ were more likely to suggest websites than those who reported $\leq 45(p<.0001)$, and nurses who reported an age of $\geq 31$ were more likely to suggest websites than those who reported an age of $\leq 30$ $(p<.0001)$. It is interesting to note that nurse age groups (age $\geq 30$ ) with formal training are less likely to recommend 
websites, while those without Internet training (age $\geq 30$ ) are more likely to make suggestions. However, the nurse age groups who use guidelines to evaluate websites (age $\geq$ 46) are more likely to make recommendations. Note, formal training referred to receiving education within an academic or a clinical in-service setting on Internet safety issues such as evaluating websites, avoiding giving out personal contact information, opening emails from an unknown source, being aware of spam, viruses, phishing, etc.

In regards to highest level of nursing degree, nurses with graduate degrees in nursing were more likely to suggest websites than those with a Bachelor's degree or lower $(p<.0001)$ Additionally, nurses who reported a nursing degree at the Bachelor's level and higher were more likely to suggest websites than those who reported an Associate degree or diploma $(p=.0029)$.

When examining one's years of experience in response to this question of suggesting websites, strong statistically significant results were found that parallel the previously noted findings with respect to age. Nurses who reported $\geq 31$ years of experience were more likely to suggest websites than those with 30 or fewer years of experience $(p=.0003)$. Nurses who reported $\geq 21$ years of experience were also more likely to make suggestions than those with 20 or fewer years of experience $(p<.0001)$. Finally respondents who reported $\geq 11$ years of experience were more likely to make suggestions than those with 10 or fewer years of experience $(p<.0001)$. This finding may suggest younger nurses may need to be encouraged to use their Internet safety training to suggest websites to their patients.

As a follow-up, this survey question also asked participants to note some of the websites they suggest to their patients. All websites with more than 10 mentions are listed in Table 4 (a steep drop off in mentions was observed for websites that received fewer than 10 mentions). It is worth noting as well that in this field, 15 nurses responded that they recommend their patients to "just surf", or to use Google or Yahoo to locate health information, while only 4 nurses responded that they instruct their patients to look for .gov, .org, and/or .edu websites for trustworthy information.

\section{Q4: Nurses offer educational content on how to assess websites}

Nurses who completed the survey were asked if they offer clients, employees, patients, and/or family members' education on how to assess if a website was safe and offered quality information. The analysis revealed no statistically significant findings with respect to age or years of experience, but there was one significant finding in relation to nurses' education level. Nurses with a Graduate degree were more likely to offer education than those with a Bachelor's degree or lower $(p=.0032)$. However, unlike the finding in the website suggestion question, it was not statistically significant for Bachelor degree or higher vs. Associate degree $(p=.3200)$.

\section{Q5: Nurses are aware of employers/organization's pol- icy regarding social media}

No statistical significance was found in relation to age, highest degree, or years of experience when nurses acknowledge they were or were not aware of their employer's/organizations policy on social media. However, nurses aged $\geq 46$ were slightly more likely to report awareness of a workplace policy on the use of the Internet and social media than those aged $\leq 45(p=.0649)$. While this result is not statistically significant under the $p<.05$ significance level, it does hint that a relationship may exist of policy awareness by older nurses. This may merit future investigation.

Table 4. Summary of healthcare websites mentioned by nurses in their survey responses

\begin{tabular}{lll}
\hline Organization & Website & \# Mentions \\
\hline WebMD & www.webmd.com & 168 \\
Centers for Disease Control and Prevention (CDC) & www.cdc.gov & 137 \\
Mayo Clinic & www.mayoclinic.org & 98 \\
American Heart Association & www.heart.org & 43 \\
American Diabetes Association & www.diabetes.org & 34 \\
National Institutes of Health & www.nih.gov & 24 \\
American Cancer Society & www.cancer.org & 17 \\
Occupational Safety and Health Administration (OSHA) & www.osha.gov & 16 \\
My Chart (refers to personalized EHR) & various & 16 \\
Medscape & www.medscape.com & 13 \\
Medline Plus & www.nlm.nih.gov/medlineplus & 13 \\
\hline
\end{tabular}


Q6: Nurse's use of the Internet or social media to extend their professional services virtually

When analyzing this question, researchers were surprised that younger nurses did not use technology and the Internet to extend their services virtually such as online diabetes educational classes, smoking cessation, and well-baby classes. Overall, only 157 (13\%) of respondents acknowledged they extended their services virtually, 887 (74\%) stated they did not, and $152(13 \%)$ offered no response. The authors believe this result may depend on the encouragement of the nurse leaders within the organization to promote this practice.

\section{Other questions reflecting no statistical significance}

The researchers found no statistical significance in regard to age, highest nursing degree achieved, or years of experience in regards to:

- Assessing clients, employees, patients, and/or their family members to determine if they use the Internet to search for health information;

- Assessing clients, employees, patients, and/or their family members to determine if the use of the Internet to search for health information is part of their organization's policy;

- Suggested websites to clients, employees, patients, and/or their family members was approved by the organization in which they were employed;

- Educational content offered by nurses on how to assess a website was approved by the organization that employs them;

- The last time nurses reviewed their organization's policy regarding use of social media.

\section{Application to practice}

Nurse leaders within various healthcare settings must support the health care needs of patients as well as their nursing professionals. By offering educational programs, developing policies, and understanding the patient as a consumer or active participant of their health needs, nurse leaders have the ability to embrace the Internet and social media to extend services virtually. A model whereby the consumer is the active participant in their health care changes the role of the consumer from being a passenger to a responsible driver of their health. ${ }^{[13]}$ In order to become active participants, consumers are becoming more knowledgeable and educated about their health care issues. Nearly $70 \%$ of consumers reported that they explored their symptoms online rather than going to the doctor first, and 64\% take steps to learn about their health condition instead of relying solely on the doctor for information. ${ }^{[14]}$

Published by Sciedu Press
This health care model may prove challenging for nurse leaders and hospital administrators. A tech-savvy patient who is knowledgeable about their healthcare will look to their providers for collaboration towards building their own virtual healthcare educational resources as well as virtual follow-up care. One way of supporting this model is to assess the patients' health information needs upon admission to the healthcare facility. Just as a detailed medical and medication history is used to guide the patient's medical care, a detailed health information-seeking assessment is needed in order to assess their knowledge and experience in retrieving health information from the Internet.

During the admission assessment, asking the consumer/ patient their desire and attitude towards learning more about their healthcare issues and their health-seeking behavior can set the foundation for developing and implementing educational interventions during hospitalization. Patients may not be aware of how to assess websites for quality information, which can be used to make critical decisions about their health. There are many patients who prefer written health information from their provider, while others see the source of health information on the Internet as a supplemental educational need. More specific questions related to their use of electronic retrieval of health information as well as questions related to computer literacy can alert the caregiver to the degree of comfort and expertise of the patient.

The importance of social media in healthcare will continue to evolve. These tech savvy patients/consumers are using social media and the Internet to obtain more health information; however the evolution of this "informed patient" will bring about far-reaching changes in the dynamics of health care and the patient-provider relationship. Providers and caregivers must be prepared to meet all of the information needs of this patient/consumer. The information requested maybe related to personal interest in clinical research studies or questions related to information within their Personal Health Record. Providers must be ready for the role the patient is actively playing in their healthcare. The face-to-cface interaction between a consumer and their healthcare provider is no longer the sole means of communication. Virtual appointments, blog posts and interactive websites allow patients to connect with online physicians and get instant information related to various medical conditions. Instant patient feedback and patient satisfactions scores will made healthcare leaders think about how to use the power of the patient to benefit all those involved in delivering high quality patient care. 


\subsection{Improving the education of nurses}

One of the challenges in meeting the consumer's educational need is meeting the educational needs of the nurses. If the nurse, as the patient educator, is not qualified to meet this educational expectation, institution-based educational programs are needed. For example, if nurses' educational need involves evaluating the Internet, the following tools could be used:

- SPAT (Site, Publisher, Author and Time), to evaluate the reliability of website content; ${ }^{[15]}$

- DISCERN, to assess the quality of written patient information on treatment choices; ${ }^{[16]}$

- The American Academy of Family Physician's Health Information on the Web; ${ }^{[17]}$

- Finding Reliable Information, the National Library of Medicine's (NLM) tutorial on Evaluating Internet Health Information ${ }^{[18]}$

The developer of the SPAT tool offers an educational guide that outlines clear direction on how to use the tool. The guide is free and available to download for personal use. ${ }^{[15]}$ Once downloaded, the guide can be shared through individual and group educational sessions to introduce how to assess websites for quality information. The DISCERN tool is a free, open source guide for healthcare professionals that offers users a valid and reliable tool to assess health information. The authors provide several additional resources to assist in educating nurses how to use the tool from general instructions, to a quick reference guide, to a list of terms, to background information on how the tool was developed. ${ }^{[16]}$ Both tools are easy to use allowing nurses to adapt quickly in using the tool.

\subsection{Policy development}

There are wonderful benefits of using social media and offering virtual services; however, correct usage is imperative. Despite nurses' age and years of experience, caregivers should be made aware of and follow institutional policy regarding the use of the Internet, social media, and virtual services. The content of these policies may include rules for:

- personal use of smartphones and computers

- maintaining patient confidentiality and privacy

- institutional approved websites and/or virtual support groups

- establishing online classes, a website

National organizations recognize the impact new electronic technologies for communication have on the healthcare industry and have developed behavioral policy examples to meet the privacy security and educational challenges organizations will encounter. One such example is Social Media in
Healthcare: Privacy and Security Considerations developed by the Hospital Information Management Systems Society (HIMSS) Privacy and Security Committee. ${ }^{[19]}$ This White Paper address not only the confidentiality and privacy issues but also the ethical issues when clinicians communicate electronically with others. The American Medical Society has also developed specific guidelines for providers when using electronic communication sites. ${ }^{[20]}$ These guidelines are not just for providers, but can be relevant for all caregivers. Nursing organizations have also developed policies that address the need for specific guidelines for employees to follow in order to maintain confidentiality for the patients entrusted to their care. ${ }^{[7,8]}$ These policies are guidelines for healthcare providers to follow in order to maintain patient confidentiality at all times whether it is in the workplace or at home.

\section{Limitations}

The researchers openly acknowledge possible limitations to the study's findings. Researchers were dependent on the five nurse leaders to disseminate the invitation for participation to all their nursing personnel. The decision to not make it mandatory for the nurses to address each question by using logic within the electronic survey, allowed nurses to skip questions they preferred not to answer. The ability for participants to move freely through the survey resulted in the total number of responses to vary per question. The length of the survey may also be considered a limitation. Asking participants to complete 51 questions in total can be a time consuming task. Finally, the validity and reliability of the questionnaire has not been tested statistically, but evaluations from several expert reviews were obtained to confirm content was appropriate. At this time, the use of the tool gave valuable data, however future testing of the tool and additional research is needed.

\section{Conclusion}

The purpose of this study was to present the findings of an exploratory study that examined nurses' use of the Internet and social media. Analysis of the findings identified a number of statistically significant correlations with respect to the age, education level, and experience of nurses. Each of these correlations provides a new avenue of inquiry into the data, new educational opportunities for health professionals and employees, as well as directing future surveys of nurses nationwide. Moving forward healthcare leaders and providers must recognize the important role social media plays in the healthcare outcomes of patients. In the era of the "engaged patient" this patient is becoming an active participant and a strong driving force in their healthcare outcomes. Their use 
of social media and technology is fueling this driving force. Healthcare leaders and providers need to recognize the role they play in the education of the patient who is using the Internet and social media sites to 1) seek health information, 2) share their health information on line, 3) virtually connect with providers and 4) ultimately revolutionize the healthcare industry.

\section{CONFLiCTS OF INTEREST Disclosure}

The authors declare that there is no conflict of interest.

\section{REFERENCES}

[1] Milton C. Ethics and social media. Nursing Science Quarterly. 2014; 27(4): 283-285. PMid:25248768 http://dx.doi.org/10.1177 /0894318414546417

[2] Jones C, Hayter M. Editorial: Social media use by nurses and midwives: A "recipe for disaster" or a "force" for good. Journal of Clinical Nursing. 2013; 22(11/12): 1495-1496. PMid:23574199 http://dx.doi.org/10.1111/jocn.12239

[3] Lachman V. Social media: Managing the ethical issues. MEDSURG Nursing. 2013; 22(5): 326-329. PMid:24358576

[4] Barrt J, Hardiker N. Advancing nursing practice through social media: A Global Perspective. Online Journal of Issues in Nursing. 2012; 17(3): 1.

[5] Spector N, Kappel D. Guidelines for using electronic and social media: The regulatory perspective. The Online Journal of Issues in Nursing. 2012; 17(3): 5.

[6] Cain J. Online social networking issues within academia and pharmacy education. American Journal of Pharmaceutical Education. 2008; 72: 1-7. http://dx.doi.org/10.5688/aj720110

[7] American Nurses Association. The ANA Principles for Social Networking and the Nurse. Silver Springs, MD, Author, 2011.

[8] National Council of State Boards of Nursing. White paper: A nurse's guide to the use of social media. 2011. https://www.ncsbn.org/ Social_Media.pdf

[9] Fox S. The social life of health information. Pew Internet \& American Life Project. 2011. http://www . pewinternet .org/ / media//Files/Reports/2011/PIP_Social_Life_of_Healt h_Info.pdf

[10] Peate I. The community nurse and the use of social media. British Journal of Community Nursing. 2013; 18(4): 180185. PMid:23574909 http://dx.doi .org/10.12968/bjcn. 201 3.18 .4 .180
[11] Douglas K. Social media: The pitfalls and the potential. Australian Nursing \& Midwifery Journal. 2014; 22(1): 24-28. PMid:25090879

[12] Bickhoff L. Smart nurses thoughtless posts on social media. Australian Nursing \& Midwifery Journal. 2014; 22(4): 31-31.

[13] Society of Participatory Medicine. Welcome. 2010; http://part icipatorymedicine.org. Accessed April 4, 2014.

[14] Lynch W, Perosino K, Slover M. Altarum Institute Survey of Consumer Health Care Opinions. 2014. Available from: http://altarum.org/sites/default/files/uploaded-r elated-files/Altarum $\% 2$ Fall $\% 202013 \% 20$ Survey $\% 20$ of $\% 2$ OConsumer\%200pinions.pdf

[15] La Rue E. What is SPAT? 2008. SPAT. Available from: http: //www.spat.pitt.edu/index.php

[16] DISCERN. General Instructions. Discern. Published n. Available from: http://www.discern.org.uk/general_instructions. php

[17] The American Academy of Family Physician's. Health Information on the Web: Finding Reliable Information. 2014. Available from: http://familydoctor.org/familydoctor/en/healthcar e-management/self-care/health-information-on-the-w eb-finding-reliable-information.htmln

[18] NLM. Evaluating Internet Health Information. 2012. Available from: http://www.nlm.nih.gov/medlineplus/webeval/we beval.html

[19] Healthcare Information Management Society. Social media in healthcare: Privacy and Security. 2013. Available from: http://himss.files.cms-plus.com/HIMSSorg/Content/f iles/Social_Media_Healthcare_WP_Final.pdf

[20] AMA. Policy Helps Guide Physicians' Use of Social Media. 2010 Available from: http://www.ama-assn.org/ama/pub/news/n ews/social-media-policy.page 